\title{
The Management of Rheumatoid Arthritis
}

\author{
Sarah Dyball, ${ }^{1}$ Anastasia-Vasiliki Madenidou, ${ }^{2}$ Christopher Saleh, ${ }^{1}$ Yvonne Tan, ${ }^{2}$ Fiona Wood, ${ }^{2}$ Lesley Ottewell ${ }^{1}$ \\ ${ }^{1}$ Rheumatology Department, RLI, ${ }^{2}$ Rheumatology Department, Furness General Hospital
}

\section{INTRODUCTION}

Rheumatoid arthritis (RA) is a chronic, systemic, inflammatory disorder of unknown aetiology that primarily involves synovial joints. If it is uncontrolled it leads to destruction of joints due to erosion of cartilage and bone, causing joint deformities. RA is the most common inflammatory arthritis affecting around 400,000 adults aged 16 and over in the UK. It has a peak onset in the 4th to 6 th decade and affects women 2 to 3 times more often. ${ }^{1,2}$

The arthritis is typically symmetrical and polyarticular and classically affects the small joints of the hands and feet leading to joint pain and swelling. Early morning stiffness is often a key feature, lasting more than 30 minutes but regularly several hours. If insufficiently treated, extraarticular manifestations may occur; the most frequent is rheumatoid nodules which are non-tender, firm subcutaneous which usually occur at bony prominences, though these can be present at onset. Due to chronic inflammation, patients with RA also have an increased risk of cardiovascular disease..$^{3,4}$

RA is a clinical diagnosis based on inflammatory arthritis, immunology tests [rheumatoid factor (RF) and anti-cyclic citrullinated peptide(anti-CCP)], acute phase reactants (erythrocyte sedimentation rate and $\mathrm{C}$ - reactive protein) and duration of symptoms more than six weeks after excluding diseases with similar clinical features.

\section{MANAGEMENT}

\section{General principles}

The treatment of RA is directed toward the control of synovitis and the prevention of joint injury. Early recognition is associated with better outcomes in patients with rheumatoid arthritis. It is recommended that patients with suspected RA should be referred to the rheumatology department as soon as possible for early initiation of DMARD therapy. Studies have shown that patients have high chances of remission if treatments are initiated within 12 weeks of a new RA diagnosis being made. ${ }^{5}$

Disease activity is measured using the DAS- 28 (disease activity score including a 28 joint count) score at baseline and during follow- up appointments (every 1-3 months). NICE guidance highlights the importance of tight control with a goal of remission or low disease activity (treat-totarget strategy). ${ }^{6}$ Once the disease is stabilised, the patient is then monitored every 6-12 months. During regular follow- ups, patients are monitored for drug toxicity and disease activity.

\section{General measures and non-pharmacologic therapies}

A number of nonpharmacologic measures and other medical interventions are important in the comprehensive management of RA in all stages of disease, in addition to antirheumatic drug therapies, such as exercise, physiotherapy, occupational therapy, cardiovascular risk reduction, bone protection and vaccinations.
Patient education and counselling are also crucial and should be commenced soon after the initial diagnosis of RA. A multidisciplinary team approach plays a key role in the management of RA. Effective communication among rheumatologists, physiotherapists, occupational therapists, pharmacists and nurses are important to ensure patient's needs are met.

Patients with persistent pain due to joint damage, worsening joint function, progressive deformity and persistent localised synovitis should be offered a referral for an early specialist surgical opinion. ${ }^{6}$

\section{Pharmacological}

Treatment of RA is a shared decision between rheumatologists and patients. The decision is individualised based on progression of disease, comorbidities and safety concerns. The first line of treatment for RA are DMARDs. These drugs can be used as monotherapy or in combination therapy. Steroids are used as short-term bridging therapy because of the slow onset of action of the DMARDs and also for exacerbations of the arthritis (known as flares).

Prior to initiation of these drugs, there are screening tests needed to be performed due to the potential side effects of the therapies. These tests may include routine bloods and also:

- Screen for Hepatitis B and C infections

- Screen for latent tuberculosis infection (for biologic drugs)

As well as a change in focus to early diagnosis with strict treatment to target guidelines, there has been a significant increase in available DMARDs due to the advances in understanding of RA pathogenesis. These are categorised broadly as synthetic or biologic. Further still, given the wealth of drug availability over the last decade these have been further subcategorised (Table 1 ). ${ }^{7}$

The introduction of biosimilar DMARDs (bsDMARDs) has also had an impact on the availability of medications for patients with RA, as well as healthcare costs. These medications must have the same activity, and demonstrate bioequivalence to the originator with at least one RCT. ${ }^{8}$ Of note, once bioequivalence has been demonstrated for one indication of the originator drug it can then be used for other originator indications. Estimates of potential savings for the United Kingdom health service are of between $£ 200$ to $£ 300$ million by $2020 / 2021$.

\section{Prognosis}

RA was previously associated with a high degree of economic loss, morbidity, and early mortality, however, impressive advances in the research associated with better understanding of the aetiopathogenesis of RA led to the discovery and therapeutic use of conventional DMARDs in 1980s and biologic DMARDs in 1990s. These therapies changed the landscape of treatment in rheumatology, leading to a real progress in achieving a better disease control.

Functional limitation, extraarticular disease, presence of RF or antiCCP and bony erosions documented 
radiographically are associated Table 1

with poorer outcomes in patients with RA. These markers of adverse prognosis can be used to identify patients who may require more aggressive pharmacotherapy, especially in early stages of disease. ${ }^{10}$

\section{CONCLUSION}

In patients with RA, affected joints may be irreversibly damaged if inflammation persists. Thus, prompt diagnosis, early recognition of active disease, and measures to quickly achieve and maintain disease control are central to modifying disease outcome. The application of these principles in the management of patients with RA, together with the development and use of newer drugs, has resulted in significant improvement in the outcomes of treatment. The discovery of new molecules and inflammatory pathways associated with disease inflammation is underway and

\begin{tabular}{|l|l|}
\hline \multicolumn{2}{|l|}{ Synthetic DMARDs (sDMARD) } \\
\hline $\begin{array}{l}\text { Conventional } \\
\text { (csDMARD) }\end{array}$ & $\begin{array}{l}\text { Targeted } \\
\text { (tsDMARD) }\end{array}$ \\
\hline $\begin{array}{l}\text { Methotrexate } \\
\text { Sulfasalazine } \\
\text { Hydroxychloroquine } \\
\text { Leflunomide }\end{array}$ & $\begin{array}{l}\text { JAK } \\
\text { inhibitors }\end{array}$ \\
\cline { 2 - 3 } Tofacitinib \\
Baricitinib
\end{tabular}

Biological DMARDs (bDMARD)

Biological (bDMARD) \& biosimilar (bsDMARD)

\begin{tabular}{|l|l|}
\hline anti-TNF drugs & $\begin{array}{l}\text { IL-6R } \\
\text { inhibitors }\end{array}$ \\
\hline Adalimumab & Tocilizumab \\
Etanercept & Sarilumab \\
Infliximab & \\
Certolizumab & \\
\cline { 1 - 1 } anti-CD20 & IL-1R \\
inhibitor \\
\hline Rituximab & Anakinra \\
\cline { 1 - 1 } anti-CD80/86 & \\
\cline { 1 - 1 } Abatacept & \\
\hline
\end{tabular}

therefore new drugs will continue to create promising opportunities for the treatment of RA.

\section{REFERENCES}

1. Goemaere S, Ackerman C, Goethals K, et al. Onset of symptoms of rheumatoid arthritis in relation to age, sex and menopausal transition. F Rheumatol 1990; 17(12): 1620-2.

2. Wasserman A. Rheumatoid Arthritis: Common Questions About Diagnosis and Management. Am Fam Physician 2018; 97(7): 455-62.

3. Avina-Zubieta JA, Choi HK, Sadatsafavi M, Etminan M, Esdaile JM, Lacaille D. Risk of cardiovascular mortality in patients with rheumatoid arthritis: a meta-analysis of observational studies. Arthritis Rheum 2008; 59(12): 1690-7.

4. Low AS, Symmons DP, Lunt M, et al. Relationship between exposure to tumour necrosis factor inhibitor therapy and incidence and severity of myocardial infarction in patients with rheumatoid arthritis. Ann Rheum Dis 2017; 76(4): 65460.

5. Monti S, Montecucco C, Bugatti S, Caporali R. Rheumatoid arthritis treatment: the earlier the better to prevent joint damage. RMD Open 2015; 1(Suppl 1): e000057.

6. Rheumatoid arthritis in adults: management NICE guideline [NG100]. https://www.nice.org.uk/guidance/ ng100/chapter/Recommendations - non-pharmacologicalmanagement (accessed 08/03/2020.

7. Mitrovic Sp, Taylor P, Fautrel B. Module 4 Treatment of Rheumatoid arthritis. Eular on- line course on Rheumatic diseases.

8. Agency EM. Guideline on similar biological medicinal products containing biotechnology-derived proteins as active substance: non-clinical and clinical issues. http:// www.ema.europa.eu/docs/en_GB/document_library/ Scientific_guideline/2015/01/WC500180219.pdf.

9. Smolen JS, Goncalves J, Quinn M, Benedetti F, Lee JY. Era of biosimilars in rheumatology: reshaping the healthcare environment. RMD Open 2019; 5(1): e000900.
10. Saag KG, Teng GG, Patkar NM, et al. American College of Rheumatology 2008 recommendations for the use of nonbiologic and biologic disease-modifying antirheumatic drugs in rheumatoid arthritis. Artbritis Rheum 2008; 59(6): 762-84.

Correspondence to: fiona.woods@mbht.nhs.uk

\section{Long Service NHS awards}

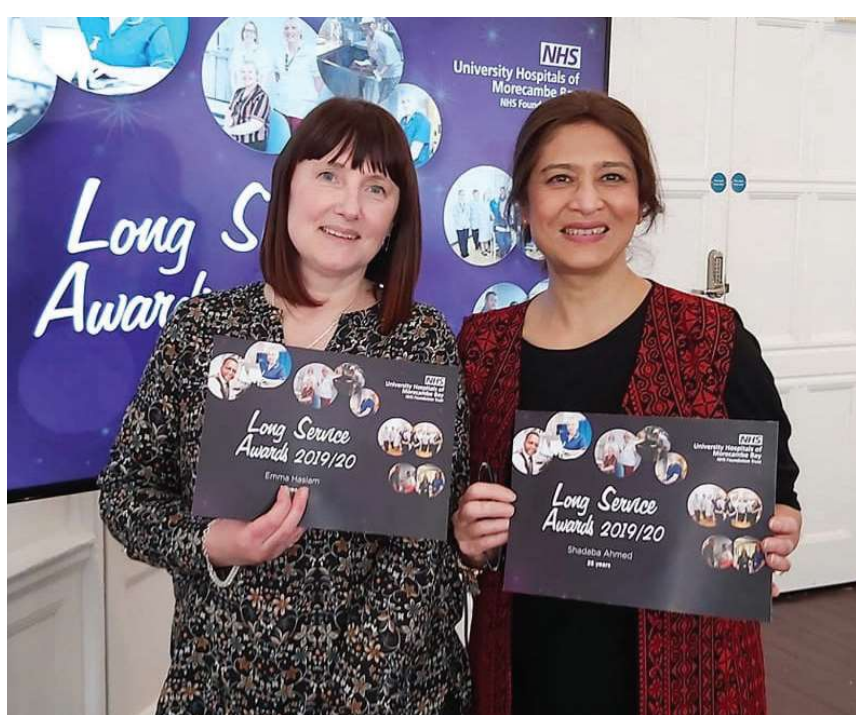

